\title{
Japon Bıldırcınlarında (Coturnix coturnix japonica) Kanatların Arterial Beslenmesi
}

\author{
Zekeriya ÖZÜDOĞRU ${ }^{1}$ Ramazan ILGÜN ${ }^{1^{*}} \quad{\text { Mehmet } \text { CAN }^{2}}^{2}$ \\ ${ }^{1}$ Aksaray Üniversitesi, Veteriner Fakültesi, Anatomi Anabilim Dall, Aksaray, Türkiye \\ ${ }^{2}$ Balıkesir Üniversitesi, Veteriner Fakültesi, Anatomi Anabilim Dall, Ballkesir, Türkiye
}

Atıf yapmak için: Özüdoğru, Z., İlgün, R. \& Can, M. (2021). Japon bıldırcınlarında (Coturnix coturnix japonica) kanatların arterial beslenmesi. Anadolu Çev. ve Hay. Dergisi, 6(4), 555-559.

How to cite: Özüdoğru, Z., İlgün, R. \& Can, M. (2021). Arterial feeding of wings in japanese quails (Coturnix coturnix japonica). J. Anatolian Env. and Anim. Sciences, 6(4), 555-559.

(iD): https://orcid.org/0000-0002-0789-3628

(iD): https://orcid.org/0000-0003-0150-3008

(D): https://orcid.org/0000-0001-9409-026X

*Sorumlu yazarın:

Ramazan İLGÜN

Aksaray Üniversitesi, Veteriner Fakültesi,

Anatomi Anabilim Dalı, Aksaray, Türkiye

凶: rilgun1980@hotmail.com
Öz: Çalışma Japon bıldırcınında kanadın arterial beslenmesini sağlayan damarların belirlenmesi amacıyla yapıldı. Araştırmada 10 adet Japon bıldırcını kullanıldı. Latex enjeksiyon metodu uygulanarak damarların seyri ve dallanmaları belirlendi. Kanatların beslenmesini sağlayan $A$. axillaris, A. subclavia'nın bir dalı olup memelilerdeki gibi devamı değildi. Omuz ekleminde $A$. subscapularis ve $A$. profunda brachii'yi verdikten sonra A. brachialis olarak seyrine devam etti. Arteria profunda brachii, $M$. teres major'un tendosu yakınında A. axillaris'ten orijin aldıktan sonra A. circumflexa ventralis humeri, A. circumflexa dorsalis humeri ve A. collateralis radialis' $\mathrm{i}$ vermekte ve A. collateralis ulnaris olarak seyrine devam ettiği tespit edilmiştir. Arteria brachialis'in orijininden sonra $M$. biceps brachii'nin caput mediale'si üzerinden geçerek dirsek eklemi hizasında a. ulnaris ve a. radialis'e ayrıldığı tespit edildi.

Anahtar kelimeler: Anatomi, bildırcın, kanat arterleri.

\section{Arterial Feeding of Wings in Japanese Quails (Coturnix coturnix japonica)}

*Corresponding author's:

Ramazan İLGÜN

Aksaray Üniversitesi, Veteriner Fakültesi,

Anatomi Anabilim Dalı, Aksaray, Turkey

凶: rilgun1980@hotmail.com

\begin{abstract}
The study was carried out to determine the arterial nourishment of the wing in Japanese quail. 10 Japanese quails were used in the study. The course and branching of the vessels were determined by applying the latex injection method. Feeding the wings A. axillaris is a branch of A. subclavia and was not a continuation of the mammals. In the shoulder joint $A$. subscapularis and A. profunda brachii after giving continued its course as brachialis. Arteria profunda brachii, near the tendo of the M. teres major after originating from A. axillaris, A. circumflexa ventralis humeri, A. circumflexa dorsalis humeri and A. collateralis radialis and it was determined that continued its course as A. collateralis ulnaris. After the origin of Arteria brachialis crossing over the $M$. biceps brachii's medial caput at the level of the elbow joint it was determined that it was devoted to $A$. ulnaris and A. radialis.
\end{abstract}

Keywords: Anatomy, quails, wing of arteries.

\section{GíRiş}

Japon bildırcını (Coturnix coturnix japonica) yüksek üreme gücü, kuluçka süresinin kısalığı, damızlıkta kullanma yaşının düşüklüğü gibi genetik faktörler ve yoğun üretim koşullarına kolay uyum sağlama yetenekleri gibi özelliklerinden dolayı yetiştiriciler ile araştırmacılar tarafindan tercih sebebi olmaktadır (Uluocak, 1991;
Yurdakul, 2006). Bıldırcın, etinin lezzeti, göğüs eti randımanının yüksekliği, et ve yumurtasının yüksek besin değeri nedeniyle günümüzde büyük bir öneme sahiptir. (Dilmen \& Özgen, 1971). Bildırcın (Coturnix coturnix) göçmen bir kuştur. Avrupa, Türkiye, Çin, Japonya ve Orta Asya olmak üzere geniş bir yaşam sahası vardır (Bariani 
vd., 2007). Ülkemizin her bölgede görülmekle birlikte, Orta Anadolu yaylalarında daha yoğun bir şekilde bulunmakta ve Trakya bölgesinde ise yerli olarak yaşamaktadırlar (Avc1, 2008; Demirsoy, 1992). Kanatlılarda dolaşım sisteminde baş, boyun, kanatlar ve göğüs bölgesini besleyen damarların kökenini aorttan çıkan truncus brachiocephalica dextra ve sinistra'dır (Nickel vd., 1977; Kuru, 1996; Dursun, 2002). Truncus brachiocephalicus'un son dallarından birisi olan A. subclavia, kanat ve pektoral bölgeyi besler (Dursun, 2002; Kuru, 1996).

A. axillaris, a. subclavia'dan çıkan bir dal olup, memelilerdeki gibi bu damarın devamı konumunda değildir (Dursun, 2002). Damar, omuz ekleminde m. teres major'un tendosunda ilk kaburga hizasında başlar ve a. subscapularis ile a. coracoidalis'i (a. subscapularis) verir daha sonra a. brachialis olarak devam eder. A. brachialis, humerus'un boyun kısmından aşar ve dirseğin distal'inden devam ederek a. radialis ve a. ulnaris isimli iki dala ayrılarak kanadın beslenmesini sağlar (Fitzgerald, 1969). $\mathrm{Bu}$ çalışmada Japon bıldırcınında kanadın arterial vaskularizasyonunu sağlayan damarlar ve verdiği dalların belirlenmesi amaçlanmıştır.

\section{MATERYAL VE METOT}

Araştırmada materyal olarak kanatlı yetiştiricilerinden temin edilen çeşitli nedenlerden ölmüş 10 adet erişkin Japon bıldırcını (Coturnix coturnix japonica) kullanılmıştır. Kloaka'dan sternum'un ucuna kadar medial bir kesit yapıldı. Daha sonra bu kesit genişletilerek vücut boşluğu ortaya çıkarılmıştır. Aorta descendens kısmı incelendi. Truncus brachiocephalicus vasıtasıyla damarlar serum fizyolojik ile yıkandı ve kırmızı kumaş boyası ile renklendirilmiş latex enjeksiyonu yapılmıştır. Latex'in pıhtılaşması için oda sıcaklığında ve çeşme suyunda bırakılan materyaller daha sonra tespit olması için \%10'luk formaldehit solusyonunda bekletilmiştir. Tespit işleminden sonra damarlar diseke edilerek fotoğraflandı. Damarların isimlerinde Nomina Anatomi Avium (Baumel vd., 1993)'daki terimler esas alınmıştır.

\section{BULGULAR}

A. axillaris (Sekil 1/1, 4/1); a.subclavia'nın bir dalı olup memelilerdeki gibi devamı değildi. Omuz ekleminde $\mathrm{m}$. teres major'un tendosunda ilk costa hizasından başladığı ve orijininden kısa bir süre sonra a. subscapularis' $i, \mathrm{~m}$. teres major'un yakınında da a. profunda brachii'yi verdikten sonra a. brachialis olarak seyrine devam ettiği belirlendi.
A. subscapularis (Şekil 1/2, 4/2): A. axillaris'in A. subclavia'dan orijin aldıktan kısa bir süre sonra çıktığı ve $M$. coracobrachialis ventralis, $M$. supracoracoideus ve M. subscapularis'i beslediği görüldü.

A. profunda brachii (Şekil 1/3, 4/31); M. teres major' un tendosunun yakınında A. axillaris'ten orijinlendi ve bu tendonun lateral'inde iki dal verdi. Bu dallardan $A$. circumflexa ventralis humeri, $M$. deltoideus ve $M$. triceps brachii'nin caput longum'u arasından dorsale doğru seyrederek bu kaslarla birlikte $M$. teres minor'a dallar verdikten sonra $M$. patagi brevis'te sonland1. Diğer dal olan A. circumflexa dorsalis humeri (Şekil 1/5, 4/6), ise $M$. latissimus dorsi' yi besleyen ramus muscularis'i (Şekil 1/8, 4/7), verdikten sonra $M$. triceps brachii'nin caput longum'u ve $M$. deltoideus arasindan oblik bir seyirle humerus'un dorsal yüzüne geçti ve dirsek ekleminde sonlandi. A. profunda brachii, humerus'un distal 1/3'ünde cranial'e doğru $A$. collateralis radialis'i verdikten sonra $A$. collateralis ulnaris olarak seyrine devam ederek dirsek ekleminin caudal'inde sonland.

A. collateralis radialis'in (Şekil 1/8): A. profunda brachii'den ayrildıktan sonra $M$. triceps brachii ve $M$. deltoideus arasından dorsal'e geçtiği ve bu bölgede $M$. extensor carpi radialis ile bölge derisini ve dirsek eklemini beslediği tespit edildi.

A. collateralis ulnaris (Şekil 1/7, 4/8): $A$. profunda brachii'nin distal devamıdı. Seyri esnasında $M$. triceps brachii ve $M$. coracobrachialis caudalis'e birçok dal verdikten sonra dirsek ekleminin caudal'inde $A$. recurrens ulnaris ile anastomozlaştı̆ı̆ gözlendi.

A. brachialis (Şekil 1/4, 2/1, 4/4): A. axillaris' in A. profunda brachialis'i verdikten sonraki distal olarak devamıdı. Damar orijininden sonra m. biceps brachii'nin caput mediale'si üzerinden geçerek distale doğru seyrine devam etti. Daha sonra M. biceps brachii'ye giden $A$. bicipitalis'i (Sekil 4/5) verdi ve dirsek eklemi hizasında $A$. ulnaris ve A. radialis'e ayrıldı.

A. axillaris'in son dallarından birisi olan $A$. ulnaris'in (Şekil 2/2), M. flexor carpi ulnaris'in cranial kenarı boyunca subcutan olarak seyrettiği ve bölge derisine A. cutanea brachialis'i verdiği tespit edildi. Damar, regio cubitalis'te $M$. flexor carpi ulnaris, M. anconeus ve adductor kasları besleyen A. recurrens ulnaris'ten (Şekil 2/10, 4/13), sonra A. ulnaris profunda'y1 (Şekil 2/4, 4/14), verdi ve a. ulnaris superficialis olarak seyrine devam etti.

A. ulnaris superficialis (Şekil 2/5, 3/1, 4/9): $A$. ulnaris'in devamı olan damar carpal eklemin distal kısminda A. metacarpalis interossea ve ramus metacarpalis ventralis'e (Şekil 3/3) ayrılarak sonlandı. Damar, seyri esnasında deri kaslarını besleyen birçok Rr. postpatagiales (Şekil 3/4, 4/16) isimli dallar verdi. 


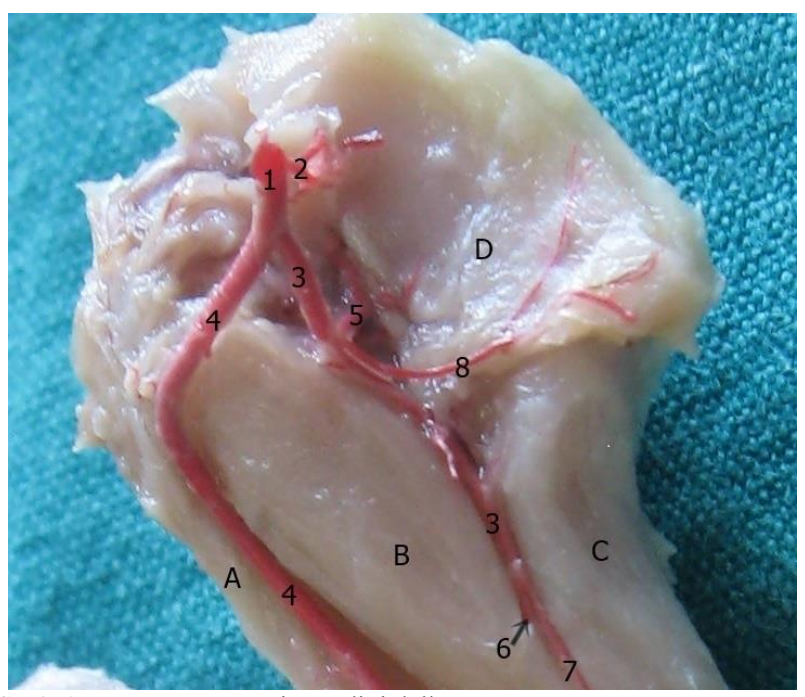

Şekil 1. Arteria axillaris'in verdiği dallar.

Figure 1. Branches given by the Arteria axillaris.

1. A. axillaris, 2. A. subscapularis, 3. A. profunda brachii, 4. A. brachialis, 5. A circumflexa dorsalis humeri, 6. A. collateralis radialis, 7. A. collateralis ulnaris, 8. Ramus muscularis, A. M. biceps brachii, B. M. triceps brachii, C. M coracobrachialis caudalis, D. M. latissimus dorsi.

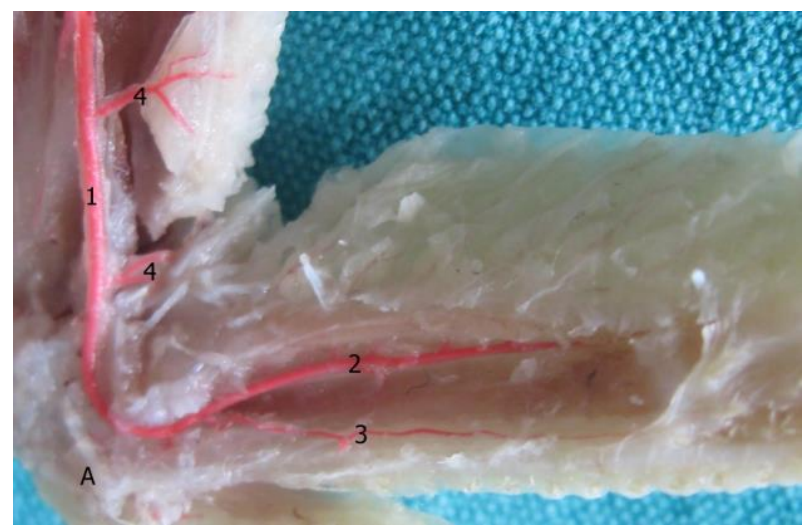

Şekil 3. Arteria ulnaris superficialis'in dalları.

Figure 3. Branches of arteria ulnaris superficialis.

1.A. ulnaris superficialis, 2. A. metacarpalis interossea, 3. Ramus metacarpalis ventralis, 4 . Rr. postpatagiales, A. Articulatio carpi.

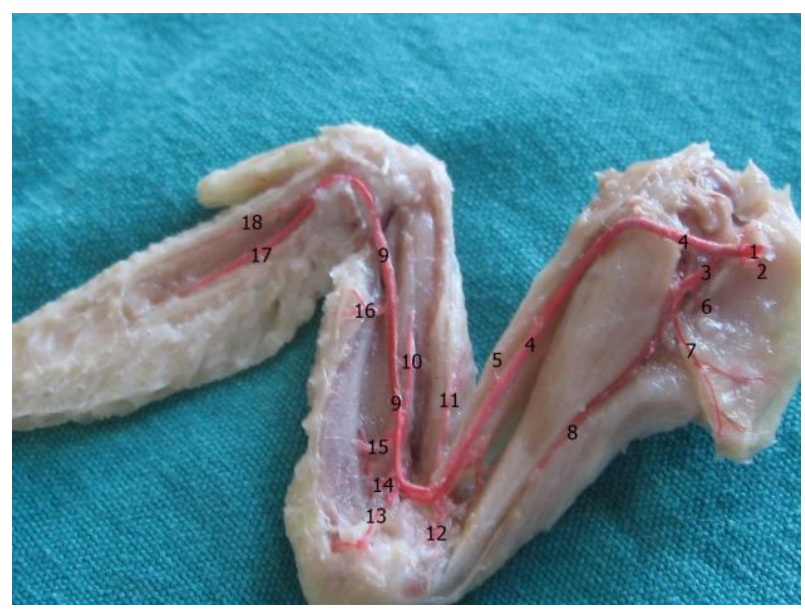

Şekil 4. Kanadı besleyen damarlar.

Figure 4. The veins that feed the wing.

1. A. axillaris, 2. A. subscapularis, 3. Arteria profunda brachii, 4. Arteria brachialis, 5. A. bicipitalis, 6. A. circumflexa dorsalis humeri, 7. Ramus muscularis, 8. A. collateralis ulnaris, 9. A. ulnaris superficialis, 10. A. radialis profunda, 11. A. radialis superficialis, 12. A. recurrens radialis, 13. A. recurrens ulnaris, 14. A. ulnaris profunda, 15. Aa. interosseus dorsales, 16. $R r$. postpatagiales, 17. A metacarpalis interossea, 18. Ramus metacarpalis ventralis.

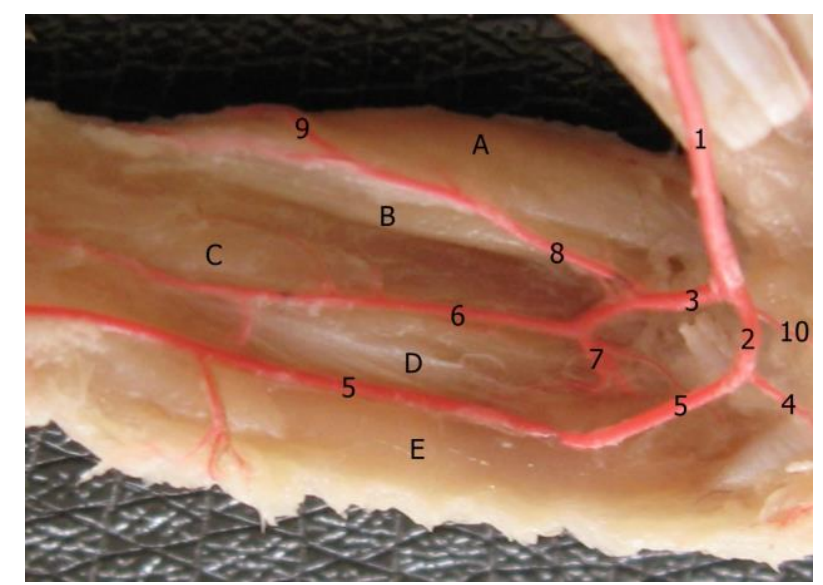

Sekil 2. Arteria brachialis ve dallanması.

Figure 2. Arteria brachialis and its branching.

1. A. brachialis, 2. A. ulnaris, 3. A. radialis, 4. A. ulnaris profunda 5. A. ulnaris superficialis, 6. A. radialis profunda, 7. Aa. interosseus dorsales, 8. A. radialis superficialis, 9. Rami propatagiales, 10. A. recurrens ulnaris, A. Musculus extensor carpi radialis, B. Musculus pronator superficialis, C. Musculus pronator profundus, D. Musculus flexor digitorum superficialis, E. Musculus flexor carpi ulnaris.

\section{TARTIŞMA VE SONUÇ}

Yapılan araştırmada birçok araştırmacının kanatlılarda (Balkaya vd., 2016; Erdoğan, 2012; Erdoğan \& Kılınç, 2014; Fisher, 1955) bildirildiği gibi a. axillaris'in a. subclavia'nın bir dalı olduğu devamı olmadığı gözlendi. Ancak bizim bulgumuzdan farklı olarak, Ali vd., (2016) tavuk, ördek ve güvercinde, El-Bably ve Rezk, (2014) tavukta, Gadhoke vd., (1975) hindide, Nora vd., (2017) kargada, Petneházy vd., (2005) güvercinde, a. axillaris'in memelilerdeki gibi A. subclavia'nın devamı olduğunu bildirmiştir.

Arteria subscapularis'in Fitzgerald, (1969) bıldırcında, Nickel vd., (1977) ve Doğuer ve Erençin, (1964) kanatlılarda bildirdikleri gibi a. axillaris'in ilk dalı olduğu ve $M$. coracobrachialis ventralis, $M$. supracoracoideus ve $M$. subscapularis'i beslediği tespit edilmiştir. Ancak bu damarın bazı turnalarda a. carotis communis'ten (Fisher, 1955), Gruiformes (Glenny, 1947) ve Galliformes (Glenny, 1951) familyalarına ait kanatlılarda ise A. cervicalis superficialis'ten köken aldığı rapor edilmiştir.

Arteria profunda brachii'nin, bildircinlarında (Fitzgerald, 1969), evcil kuşlarda (Doguer \& Erencin, 1964) ve atmacada (Balkaya vd., 2016) A. axillaris'ten orijin aldığı ve $M$. triceps brachii'nin (Nickel vd., 1977) caput longum'unu beslediği bildirilmiştir. Benzer şekilde A. profunda brachii'nin $M$. teres major'un tendosunun yakınında $A$. axillaris'ten orijin aldığ ventralis humeri, A. circumflexa dorsalis humeri, A. collateralis radialis'i verdikten sonra $A$. collateralis ulnaris olarak seyrine devam ettiği ve dirsek ekleminde sonlandığ́ gözlenmiştir. 
Yapılan çalışmada, A. brachialis'in literatürlerde (Arad vd., 1989; Bezuidenhout \& Coetzer, 1986; Gobeil, 1970; Nickel vd., 1977) bildirdiği gibi A. axillaris'in distal devamı olduğu ve $A$. ulnaris ile $A$. radialis'e ayrıldığı tespit edilmiştir.

Doğuer ve Erencin (1964)'ün evcil kanatlılardaki bildirdiklerinin aksine, bu çalışmada arteria brachialis'in $A$. ulnaris ve $A$. radialis'e ayrıldı̆̆ ortasında değil de dirsek eklemi hizasında olduğu tespit edilmiştir.

Sunulan bu çalışmada, A. ulnaris profunda'nın zayıf bir damar olduğu ve A. ulnaris'ten tek bir damar olarak çıktığ 1 tespit edilmiştir. Bu tespitimizle ilgili olarak Baumel vd., (1993) tavuk ve güvercinde, Balkaya vd., (2016), atmacada, Nora vd., (2017) kargada adı geçen damarın zayıf bir arter olduğu bildirmişler ancak, Balkaya vd., (2016) atmacada A. ulnaris profunda'nın A. recurrens ulnaris ile ortak bir kökten çıktığını rapor etmiştir. A. ulnaris'in, Balkaya vd., (2016) atmacada bildirdiği gibi articulatio carpi'nin distal k1sminda Arteria metacarpalis interossea ve Ramus metacarpalis ventralis'e ayrilarak sonlandığı tespit edilmiştir.

Sonuç olarak; bıldırcınlarda a. axillaris'in dallanmasının diğer kuşlarla benzer olduğu tespit edilmiştir. Oluşan bazı farklılıkların ise tür farkından veya damar sisteminde görülen varyasyonlardan kaynaklandığ sonucuna varılmıştır.

\section{KAYNAKLAR}

Abdel-Khalek, A.A., Kadry, M.A.M., Badran, S.R. \& Marie, M.S. (2015). Comparative Toxicity of Copper Oxide Bulk and Nano Particles in Nile Tilapia; Oreochromis niloticus: Biochemical and Oxidative Stress. Journal of Basic and Applied Biology, 72, 43-57. DOI: 10.1016/j.jobaz.2015.04.001

Arad, Z., Midtgard, U. \& Bernstein, M.H. (1989). Thermoregulation in Turkey vultures. vascular anatomy, arteriovenous heat exchange, and behavior. Condor, 91(3), 505-514. DOI: $10.2307 / 1368103$

Avcı, S. (2008). Coturnix coturnix L., 1758 ve Alectoris chukar Gray, 1830 türlerinin karyolojik özellikleri. Yüksek Lisans Tezi, Sakarya Üniversitesi Fen Bilimleri Enstitüsü, Biyoloji Anabilim Dalı, Sakarya, Türkiye.

Balkaya, H., Özüdoğru, Z., Özdemir, D., Can, M., Erbas, E. \& Kara, H. (2016). The vascular arrangement and main branches of arteria axillaris in wing of sparrowhawk (Accipiter nisus.). Journal of Hellenic Veterinary Medicine Society, 67(3), 171-176. DOI: 10.12681/jhvms.15635

Bariani, M., Bernard-Laurent, A., Mucci, N., Tabarroni, C., Kark, S., Garridoe, J.A.P. \& Randi, A. (2007). Hybridisation with introduced chukars (Alectoris chukar) threatens the gene pool integrity of native rock (A. graeca) and red legged (A. rufa) partridge populations. Biological Conservation, 137, 57-69. DOI: 10.1016/j.biocon.2007.01.014

Baumel, J.J., King, S.A., Breazile, J.E., Evans, H.E. \& Vanden Berge, J.C. (1993). Handbook of Avian Anatomy. Nomina Anatomica Avium. 2. Ed. Published By the Club, 469-481, Cambridge, Massachusetts.

Bezuidenhout, A.J. \& Coetzer, D.J. (1986). The major blood vessels of the wing of the ostrich (Struthio camelus). Onderstepoort Journal of Veterinary Research, 53(4), 201-203.

Demirsoy, A. (1992). Yaşamın Temel Kuralları. omurgalılar/amniyota (sürüngenler, kuşlar ve memeliler). Meteksan Yayınevi, Ankara.

Dilmen, S. \& Özgen, H. (1971). Yeni bir protein kaynă̆ bıldircın, 280, Ankara Üniversitesi Veteriner Fakültesi Yayınları, Türkiye.

Doğuer, S. \& Erençin, Z. (1964) Evcil kuslarin komparatif Anatomisi. Ankara Üniversitesi Veteriner Fakültesi Yayınları: 176, 68-77. Ankara Üniversitesi Basımevi, Ankara.

Dursun, N. (2002). Evcil kuşların anatomisi, Medisan yayınevi 1. bask1, Ankara.

El-Bably, S.H. \& Rezk, H.M. (2014). The arterial vasculature of the wing in domestic fowl (Gallus gallus domesticus). Veterinary Medical Journal, 60, 105-123.

Erdoğan, S. \& Kılınç, M. (2014). The branching pattern of the aortic arch in the long-legged buzzard (Buteo rufinus, Cretzschmar 1829). Anatomical Science International 89:151-155. DOI: 10.1007/s 12565-013-0210-1

Erdoğan, S. (2012). The branching of the aortic arch in the Eurasian bittern (Botaurus stellaris, Linnaeus 1758). Veterinarni Medicina Journal, 57(5), 239244.

Fisher, H.I. (1955). Major arteries near the heart in the whooping crane. Condor, 57, 286-289.

Fitzgerald, T.C. (1969). The coturnix quail, qnatomy and histology. 195-200. The Iowa State University Press, Ames, Iowa.

Gadhoke, R.T., Lindsay, R.T. \& Desmond, R.T. (1975). Comparative study of the blood vascular system of the cetvico-thoracic region and thoracic limb of the domestic Turkey (Meleagris gallopavo). Anatomischer Anzeiger, 138, 39-45.

Glenny, F.H. (1947). A Systematic Study of the Main Arteries in the Region of the Heart-Aves XIV Gruiformes, Part 2. Auk, 64(3), 407-410.

Glenny, F.H. (1951). A Systematic Study of the Main Arteries in the Region of the Heart-Aves XII Galliformes, Part 1. The Ohio Journal of Science, 51(1), 47-54.

Gobeil, R.E. (1970). Arterial system of the Herring gull (Larus argentatus). Journal of Zoology, 160(3), 337-354.

Kuru, N. (1996). Evcil tavuk ve yeni zelanda tavşanında aorta'nın seyri ve dağılımı üzerinde 
makroanatomik araştırmalar. Selçuk Üniversitesi Fen Bilimleri Enstitüsü, Konya, 1996.

Nickel, R., Schummer, A. \& Seifirle, E. (1977). Anatomy of the Domestic Birds, 131-139. Verlag Paul Parey, Berlin, Hamburg.

Nora, A., Shaker \& Nawal, A.N. (2017). Morphological features of the bones and the arterial supply of the wing in the hooded crow (corvus cornix) with special reference to the muscles of flight. International Journal of Veterinary Science, 6(2), 96-103.

Petneházy, Ö., Sótonyi, P. \& Tóth, M. (2005). Blood supply to the pigeon wing and flying muscles. Anatomia, Histologia, Embryologia, 34, 39. DOI: 10.1111/j.1439-0264.2005.00669_88.x

Safwat, A., Mona A.N. \& Eresha, M.A. (2016). Macro and micro architecture of the wing in three different avian habitats. Alexandria Journal of Veterinary Sciences, 48, 134-142. DOI: 10.1111/j.1096-3642.1990.tb01865.x

Uluocak, A.N. (1991). Çukurova'da hayvansal üretimde yeni bir kaynak, bıldırcın. Çukurova Üniversitesi Ziraat Fakültesi I. Çukurova Tarım Kongresi, 422-427. Adana.

Yurdakul, F. (2006). Bildircinlarda bireysel ve grup düzeyinde erkek katımında erkek/dişi oranlarının döllülüğe etkisi. Yüksek Lisans Tezi,Çukurova Üniversitesi Fen Bilimleri Enstitüsü, Zootekni Anabilim Dal, Adana. 